\title{
Impact of Heat and Mass Transfer on MHD Oscillatory Flow of Jeffery Fluid in a Porous Channel with Thermal Conductivity, Dufour and Soret
}

\author{
*11IDOWU, AS; ${ }^{2}$ JIMOH, A.; ${ }^{3}$ AHMED, LO
}

\author{
Department of Mathematics University of Ilorin, Ilorin, Nigeria. \\ Hassan Usman Katsina Polytechnic Katsina ,Katsina, Nigeria. \\ *EMAIL of corresponding author: asidowu@gmail.com,abdulwaheedjimoh@gmail.com
}

\begin{abstract}
The objective of this paper is to study Dufour, Soret and thermal conductivity on unsteady heat and mass transfer of magneto hydrodynamic (MHD) oscillatory flow of Jeffery fluid through a porous medium in a channel. The partial differential equations governing the flow have been solved numerically using semi-implicit finite-difference scheme with the aid of MATLAB software. The results obtained are displayed graphically and in tabular form to illustrate the effect of various parameters on the dimensionless velocity, temperature and concentration profiles, to show the effects of different parameters entering in the problem. Results from these study shows that velocity and temperature increases with the increase of Soret and Dufour why the thermal conductivity increases as temperature profile increasing. Also, it is observed that soret number increases as concentration profile decreases. (O) JASEM http://dx.doi.org/10.4314/jasem.v19i4.32
\end{abstract}

KEY WORDS: Heat and Mass Transfer, Dufour, Soret, Jeffery Fluid and Thermal Conductivity

\section{INTRODUCTION}

The Jeffery fluid flow with heat and mass transfer problem have many practical applications in sciences and technology. It is used in agricultural engineering to study the underground water resources, seepage of water in river-beds, food processing, magneto hydrodynamics (MHD) generators, geothermal energy extraction, electromagnetic propulsion, polymer solution and the boundary layer control in the field of aerodynamics and so on. The combined heat and mass transfer has many applications in difference branches of science and technology. The influence of heat transfer on MHD oscillatory flow of Jeffery fluid in a channel has been done by Kavita et .al. (2013). The problem was solved analytically using perturbation technique and the solution obtained shows that a rise in the chemical reaction parameter leads to reduction of the velocity as well as species concentration.

Vidyasadar and Ramana (2013), investigated the radiation effect on MHD free convection flow of Kuvshinshiki fluid with mass transfer past a vertical porous plate through porous medium. The solution to the problem were obtained by using the Perturbation technique, it is observed that the velocity decreases as visco-elastic increases. Idowu et al. (2013) also investigated the effect of heat and mass transfer on unsteady MHD oscillatory flow of Jeffery fluid in a horizontal channel with chemical reaction. The significant of their study revealed that the velocity is more of Jeffery fluid than that of Newtonian fluid.
Srinivasa et al. (2013), worked on effect of heat transfer on MHD oscillatory flow of Jeffery fluid through a porous medium in a tube. The governing partial differential equation have been solved by using perturbation technique, it is found that, the velocity is more for Jeffery fluid than that of Newtonian fluid. Finite element solution of heat and mass transfer in MHD flow of a viscous fluid past vertical porous plate under oscillatory suction velocity has been discussed by Anand Rao et al. (2012), The problem has being solved by Galerking finite element method. It was found that the increase in magnetic field contributes to the decrease in the velocity field.

The importance of Soret (thermal -diffusion) and Dufor (diffusion thermo) as it influenced the fluids with very light molecular weight as well as medium molecular weight have received attention by researchers and useful results have been reported. Bhupendra K. Sharma, Kailash Yadav Nidhish K. Mishra et al. (2012), considered the Soret and Dufor effect on unsteady MHD mixed convection flow past a radiative vertical porous plate embedded in a porous medium with a chemical reaction. The problems were solved numerically applying explicit finite difference method, the solution obtained shows that the fluids with medium molecular weight (H2, air), Dufor and Soret effect should not be neglected. 
Bhavana et al. (2013), presented the soret effect on free convective unsteady MHD flow over a vertical plate with heat source. The governing equation for this problem are solved analytically by using perturbation method, the result obtained shows that the velocity profile increase with an increasing of soret number, i.e. the fluid velocity raises due to greater in thermal diffusion. Anuradha et al (2014), in their work, studied the Heat and mass transfer on unsteady MHD free convective flow past a semi-infinite vertical plate with soret effect. The problem was investigated for both aiding and opposing flow using perturbation technique and their result shows that the soret number increases as the velocity profile increases. Uwanta and Omokhuale (2014), considered the effects variable thermal conductivity on heat and mass transfer with Jeffery fluid.

Hence, in this present study, special attention is given to heat and mass transfer on magnetohydro-dynamics
(MHD) oscillatory flow of Jeffery fluid through a porous medium in a channel in the presence of thermal radiation, thermal conductivity, Dufour and Soret. The expressions are obtained for velocity, temperature and concentration numerically. The influence of various parameters on the velocity, temperature and concentration are discussed in detail through several graphs and tables.

Mathematical Formulation.: We considered the onedimensional free convective and mass transfer flow of an incompressible Jeffrey fluid in a channel of width $h$ under the influence of electrically applied magnet field and radiative heat transfer as shown in the figure below. It is assumed that the fluid has very small electrical conductivity and electromagnetic force respectively. We choose the Cartesian coordinate system $(\mathrm{x}, \mathrm{y})$, where $\mathrm{x}$ - is taken along center of the channel and $\mathrm{y}$ - axis is taken normal to the flow direction.

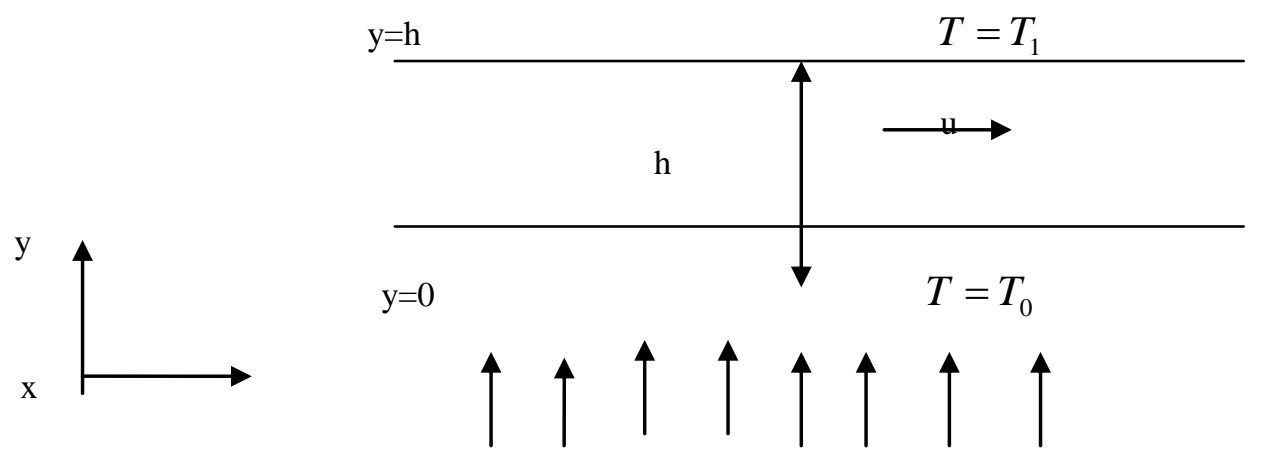

Flow Configuration and coordinate system of the Problem.

Hence, $\quad S=\frac{\mu}{1+\lambda_{1}}\left(\frac{\partial \gamma}{\partial t^{*}}+\lambda_{2} \frac{\partial \gamma}{\partial t^{* 2}}\right)$

Where, $\mu$ is the dynamic viscousity, $\lambda_{1}$ is the ratio of relaxation to retardation times, $\lambda_{2}$ is the retardation time and $\frac{\partial \gamma}{\partial t^{*}}$ is the shear rate.

The basic equations of momentum and energy governing such a flow, subject to the Boussinesq approximation, are:

Continuity equation

$\frac{\partial u}{\partial y}=0$

Momentum equation:

$$
\frac{\partial u^{\prime}}{\partial t^{\prime}}+v^{\prime} \frac{\partial u^{\prime}}{\partial y^{\prime}}=-\frac{\partial P}{\partial x}+\frac{\mu}{1+\lambda 1} \frac{\partial^{2} u^{\prime}}{\partial y^{\prime 2}}+g \beta\left(T^{\prime}-T_{\infty}^{\prime}\right)+g \beta\left(C^{\prime}-C_{\infty}^{\prime}\right)+\frac{\sigma B_{0}^{2}}{\rho} u^{\prime}+\frac{v}{k^{\prime}} u^{\prime}
$$


Temperature equation:

$$
\frac{\partial T^{\prime}}{\partial t^{\prime}}+v^{\prime} \frac{\partial T^{\prime}}{\partial y^{\prime}}=\frac{k_{0}}{\rho C p} \frac{\partial}{\partial y^{\prime}}\left(K(T) \frac{\partial T^{\prime}}{\partial y^{\prime}}\right)-\frac{1}{\rho C p} \frac{\partial q_{r}}{\partial y^{\prime}}-\frac{Q_{0}}{\rho C p}\left(T^{\prime}-T_{\infty}^{\prime}\right)+\frac{v}{C_{\rho}}\left(\frac{\partial u^{\prime}}{\partial y^{\prime}}\right)^{2}+\frac{D_{M}}{\rho C \rho} \frac{\partial^{2} \varphi}{\partial y^{\prime 2}}(3)
$$

Concentration equation

$$
\frac{\partial C^{\prime}}{\partial t^{\prime}}+v^{\prime} \frac{\partial C^{\prime}}{\partial y^{\prime}}=D m \frac{\partial^{2} C^{\prime}}{\partial y^{\prime 2}}+\frac{D_{m} K_{T}}{T_{m}} \frac{\partial^{2} T^{\prime}}{\partial y^{\prime 2}}-K_{r}\left(C^{\prime}-C_{\infty}^{\prime}\right)
$$

The boundary conditions are

$$
\begin{aligned}
& u=0, \quad T=0, \quad C=0 \quad \text { at } \quad \mathrm{y}=0 \\
& u=0, \quad T=T_{1}, \quad C=C_{1} \quad \text { at } \quad \mathrm{y}=\mathrm{h}
\end{aligned}
$$

Where $\mathrm{u}$ is the axial velocity, $\mathrm{T}$ is the fluid temperature, $\mathrm{P}$ is the pressure, $\rho$ is the fluid density, $B_{0}$ and is the magnetic field strength, $\sigma$ is the conductivity of the fluid, $\mathrm{g}$ is the acceleration due to gravity, $\beta$ is the coefficient of volume expansion due to temperature, $C p$ is the specific heat at constant pressure.k is the thermal conductivity and $\mathrm{q}$ is the radiative heat flux, $D m$ is the coefficient of mass diffusivity, $K_{T}$ is the thermal diffusion ratio, $m$ is a constant, $T_{m}$ is the mean fluid temperature and $K_{r}$ is the chemical reaction, $\mathrm{qr}$ is the radiation heat flux, $v$ is the kinematic viscosity and subscript $\infty$ denotes the conditions in the free stream.

For optically thick fluid we have modest (1993) as follows radiative heat flux term by using the Rosseland approximation given by;

$q_{r}^{\prime}=\frac{4 \sigma^{\prime}}{3 k_{r}^{\prime}} \frac{\partial T^{\prime 4}}{\partial y^{\prime}}$

Where $\sigma$ is the Stelfan-Boltzmann constant? It should be noted that by using the Rosseland approximation, the present analysis is limited to optically thick fluids. If the temperature differences within the flow are sufficient small, then equation (7) can be literalized by expanding $T^{\prime 4}$ into the Taylor series about $T_{\infty}^{\prime}$, which after neglecting higher order terms take the form

$$
\begin{gathered}
T^{\prime 4} \cong 4 T_{\infty}^{\prime 3} T^{\prime}-3 T_{\infty}^{\prime 4} \\
q_{r}^{\prime}=-\left(\frac{16 \sigma T_{\infty}^{\prime}}{3 k_{r}^{\prime}}\right)
\end{gathered}
$$

Substituting (8) and (9), into equation (3) we have

$$
\frac{\partial T^{\prime}}{\partial t^{\prime}}+v^{\prime} \frac{\partial T^{\prime}}{\partial y^{\prime}}=\frac{k_{0}}{\rho C p} \frac{\partial}{\partial y^{\prime}}\left(K(T) \frac{\partial T^{\prime}}{\partial y^{\prime}}\right)+\frac{16 \sigma s}{3 \rho C p k e} T_{\infty}^{\prime 3} \frac{\partial^{2} T^{\prime}}{\partial y^{\prime 2}}
$$

The thermal conductivity depends on temperature. It is used by molla et al. (2005), as follows

$$
K(T)=k_{0}\left\{1+m\left(T^{\prime}-T_{\infty}^{\prime}\right)\right\}
$$

Where $k_{0}$ is the thermal conductivity of the ambient fluid and $\tau$ is defined by

$$
\text { *IIDOWU, AS; }{ }^{2} \text { JIMOH, A.; }{ }^{3} \text { AHMED, LO }
$$




$$
\tau=\frac{1}{K(T)}\left(\frac{\partial K(T)}{\partial T}\right)
$$

$v_{0}>0$ is the suction parameter and $v_{0}<0$ is the injection parameter. On introducing the following nondimensional variables

$$
\begin{aligned}
& u=\frac{u^{\prime}}{U_{0}}, y=\frac{y^{\prime}}{H}, t=\frac{t^{\prime} U_{0}}{H}, \theta=\frac{T^{\prime}-T_{\infty}^{\prime}}{T_{w}^{\prime}-T_{\infty}^{\prime}}, \varphi=\frac{C^{\prime}-C_{\infty}^{\prime}}{C_{w}^{\prime}-C_{\infty}^{\prime}}, \operatorname{Pr}=\frac{U_{0} \rho C \rho}{k_{0}}=\frac{v}{\alpha}, G r=\frac{g \beta H\left(T^{\prime}-T_{\infty}^{\prime}\right)}{U_{0}^{2}}, \\
& G c=\frac{g \beta H\left(C^{\prime}-C_{\infty}^{\prime}\right)}{U_{0}^{2}}, M=\frac{\sigma B_{0}^{2} H}{\rho U_{0}^{2}}, R=\frac{4 \sigma T_{\infty}^{\prime 3}}{k k_{e}}, S c=\frac{U_{0}}{D}, \\
& S r=\frac{D m K_{T}\left(T^{\prime}-T_{\infty}^{\prime}\right)}{T_{m} v\left(\varphi^{\prime}-\varphi_{\infty}^{\prime}\right)}, D u=\frac{D M\left(\varphi^{\prime}-\varphi_{\infty}^{\prime}\right)}{u_{0} \rho C \rho\left(T^{\prime}-T_{\infty}^{\prime}\right)}, K \varphi=\frac{k_{r} H}{U_{0}}, \gamma=\frac{v_{0} H}{U_{0}}, \tau=m=\left(T^{\prime}-T_{\infty}^{\prime}\right), \\
& K=\frac{k^{\prime} U_{0}}{v H}, \quad \mathrm{Q}=\frac{H Q_{0}}{\rho C \rho U_{0}},
\end{aligned}
$$

where $\mathrm{U}$ is the mean flow velocity, into the equations (2), (3) and (4) with equation (1) identically satisfied the following set of differential equations, (after dropping bars)

$$
\begin{aligned}
& \frac{1}{4} \frac{\partial u}{\partial t}-\left(1+A \varepsilon e^{i \omega t}\right) \frac{\partial u}{\partial y}=-\lambda e^{i \omega t}+\frac{1}{1+\lambda 1} \frac{\partial^{2} u}{\partial y^{2}}+G r \theta+G c \varphi-\left(M+\frac{1}{K}\right) u \sim \sim \sim \sim \sim ~(12) \\
& \frac{1}{4} \frac{\partial \theta}{\partial t}-\left(1+A \varepsilon e^{i \omega t}\right) \frac{\partial \theta}{\partial y}=\frac{\lambda}{\operatorname{Pr}}\left(\frac{\partial \theta}{\partial y}\right)^{2}+\frac{1}{\operatorname{Pr}}(1+\tau \theta) \frac{\partial^{2} \theta}{\partial y^{2}}+\operatorname{Ec}\left(\frac{\partial u}{\partial y}\right)^{2}-(Q+R) \theta+\operatorname{Du} \frac{\partial^{2} \varphi}{\partial y^{2}}
\end{aligned}
$$

13)

$$
\frac{1}{4} \frac{\partial \varphi}{\partial t}-\left(1+A \varepsilon e^{i \omega t}\right) \frac{\partial \varphi}{\partial y}=\frac{1}{S c} \frac{\partial^{2} \varphi}{\partial y^{2}}+S r \frac{\partial^{2} \theta}{\partial y^{2}}-\operatorname{Kr} \varphi
$$

Where $\mathrm{M}$ is the Hartman number, $\mathrm{Gr}$ is the Grashof number, Pr is Prandlt number and $\mathrm{R}$ is the radiation parameter.

Numerical Solution Procedure: The set of couple nonlinear governing differential equations (12)-(14) together with initial and boundary conditions (24) and (25) are solved numerically by using the implicit finite difference technique of Crank-Nicolson type which is known to converge rapidly. The finite difference approximations equivalent to (12)-(24) using the method are as follows. 


$$
\begin{aligned}
& \left(\frac{u_{i}^{j+1}-u_{i}^{j}}{4 \Delta t}\right)-\frac{B}{2 \Delta y}\left(u_{i+1}^{j}-u_{i-1}^{j}\right)=-\lambda H P 1+\frac{1}{2(\Delta y)^{2}}\left(u_{i+1}^{j+1}-2 u_{i}^{j+1}+u_{i-1}^{j+1}+u_{i+1}^{j}-2 u_{i}^{j}+u_{i-1}^{j}\right)-\left(M+\frac{1}{K}\right) u_{i}^{j} \\
& +\operatorname{Gr}(\theta)_{i}^{j}+G(\varphi)_{i}^{j} \\
& \left(\frac{\theta_{i}^{j+1}-\theta_{i}^{j}}{4 \Delta t}\right)-\frac{B}{2 \Delta y}\left(\theta_{i+1}^{j}-\theta_{i-1}^{j}\right)=\frac{F}{2(\Delta y)^{2}}\left(\theta_{i+1}^{j+1}-2 \theta_{i}^{j+1}+\theta_{i-1}^{j+1}+\theta_{i+1}^{j}-2 \theta_{i}^{j}+\theta_{i-1}^{j}\right)+\frac{\tau}{2 \operatorname{Pr}(\Delta y)^{2}}\left(\theta_{i+1}^{j+1}-\theta_{i}^{j}\right)^{2} \\
& -(Q+R)(\theta)_{i}^{j}+\frac{D u}{2(\Delta y)^{2}}\left(\varphi_{i+1}^{j+1}-2 \varphi_{i}^{j+1}+\varphi_{i-1}^{j+1}+\varphi_{i+1}^{j}-2 \varphi_{i}^{j}+\varphi_{i-1}^{j}\right)+\frac{E c}{(\Delta y)^{2}}\left(u_{i+1}^{j+1}-u_{i}^{j}\right)^{2}, \\
& \left(\frac{\varphi_{i}^{j+1}-\varphi_{i}^{j}}{4 \Delta t}\right)-\frac{B}{2 \Delta y}\left(\varphi_{i+1}^{j}-\varphi_{i-1}^{j}\right)=\frac{1}{2 S c(\Delta y)^{2}}\left(\varphi_{i+1}^{j+1}-2 \varphi_{i}^{j+1}+\varphi_{i-1}^{j+1}+\varphi_{i+1}^{j}-2 \varphi_{i}^{j}+\varphi_{i-1}^{j}\right)+ \\
& \frac{S r}{2(\Delta y)^{2}}\left(\theta_{i+1}^{j+1}-2 \theta_{i}^{j+1}+\theta_{i-1}^{j+1}+\theta_{i+1}^{j}-2 \theta_{i}^{j}+\theta_{i-1}^{j}\right)-K r \text {. }
\end{aligned}
$$

The initial and boundary conditions may be expressed as

$$
\begin{array}{lllll}
u_{i, j}=0, & \theta_{i, j}=0, & \varphi_{i, j}=0 & & \\
u_{y, j}=0, & \theta_{y, j}=1, & \varphi_{y, j}=1 & \text { at } & \mathrm{y}=0 \\
u_{y, j}=0, & \theta_{y, j}=0, & \varphi_{y, j}=0 & \text { at } & \mathrm{y}=1
\end{array}
$$

Where $\mathrm{B}=\left(1+\mathrm{A} \varepsilon \mathrm{e}^{(\omega i t)}\right), \quad \mathrm{P} 1=(1+\lambda 1), \mathrm{H}=\exp (\omega i t), \quad \mathrm{F}=(1+\tau \theta)$

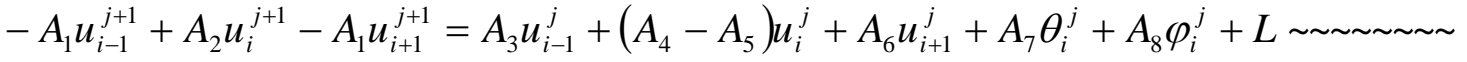

$$
\begin{aligned}
& -B_{1} \theta_{i-1}^{j+1}+B_{2} \theta_{i}^{j+1}-B_{1} \theta_{i+1}^{j+1}=B_{3} \theta_{i-1}^{j}+B_{4} \theta_{i}^{j}+B_{5} \theta_{i+1}^{j}+B_{6}\left(\theta_{i+1}^{j}-\theta_{i}^{j}\right)^{2}+B_{7}\left(u_{i+1}^{j}-u_{i}^{j}\right)^{2} \\
& +B_{8}\left(\varphi_{i+1}^{j+1}-2 \varphi_{i}^{j+1}+\varphi_{i-1}^{j+1}+\varphi_{i+1}^{j}-2 \varphi_{i}^{j}+\varphi_{i-1}^{j}\right) \sim \sim \\
& -D_{1} \varphi_{i-1}^{j+1}+D_{2} \varphi_{i}^{j+1}-D_{1} \varphi_{i+1}^{j+1}=D_{3} \varphi_{i-1}^{j}+D_{4} \varphi_{i}^{j}+D_{5} \varphi_{i+1}^{j}-D_{6} \varphi_{i}^{j} \\
& +D_{7}\left(\theta_{i+1}^{j+1}-2 \theta_{i}^{j+1}+\theta_{i-1}^{j+1}+\theta_{i+1}^{j}-2 \theta_{i}^{j}+\theta_{i-1}^{j}\right) \\
& C_{f}=-\left(\frac{\partial u}{\partial y}\right)_{y=0}, \quad N u=-\left(\frac{\partial \theta}{\partial y}\right)_{y=0}, \quad S h=-\left(\frac{\partial \varphi}{\partial y}\right)_{y=0}
\end{aligned}
$$

The index I corresponds to space $\mathrm{y}$ and $\mathrm{j}$ corresponds to time t. $\Delta y$ and $\Delta t$ are the mesh sizes along $\mathrm{y}$ direction and time t-direction respectively. The finite difference equations (19)-(21) at every internal nodal point on a particular n-level constitute a tri-diagonal system of equations, which are solved by using the Thomas algorithm.

During the computation in each time step, the temperature and concentration profile have been computed from equation (19) and (20) and then the computed values are used to obtain the velocity profile at the end of time

Steps that is $u_{i, j+1}$ computed from equation (19). Computations are carried out until the steady state is reached. The steady-state solution of the convergence criteria for stability of the scheme is assumed to have been reached, when the absolute differences between the value of velocity, temperature and concentration at two consecutive time steps are less than $10^{-5}$ at all grid points. Computations are carried out for different values of physical parameters involved in the problem.

\section{RESULTS AND DISCUSSION}

Numerical evaluation for the solution of this problem is performed and the results are illustrated graphically in Figs. 1-20. This results show the influence of the various physical parameters such as Grashof number Gr, Solutal Grashof number G $\varphi$,Magnetic parameter M, Permeability parameter $\mathrm{K}$, dimensionless heat source coefficient Q, Prandtl number Pr, Dufour number $\mathrm{Du}$, Soret number $\mathrm{Sr}$, Chemical reaction $\mathrm{Kr}$, 
Schmidt number Sc, Ekert number Ec, Radiation parameter $\mathrm{R}$, Thermal conductivity parameter $\tau, \lambda_{1}$ is the ratio of relaxation to retardation times, $\lambda$ is the real constant and $\omega$ is the frequency of the oscillation. We have also analyzed the effects of these physical parameters on Skin friction coefficient, Nusselt number and Sherwood number distribution for

\section{Velocity Profiles}

In Figures.1-10, we presented the behavior of the fluid velocity for various material parameters.

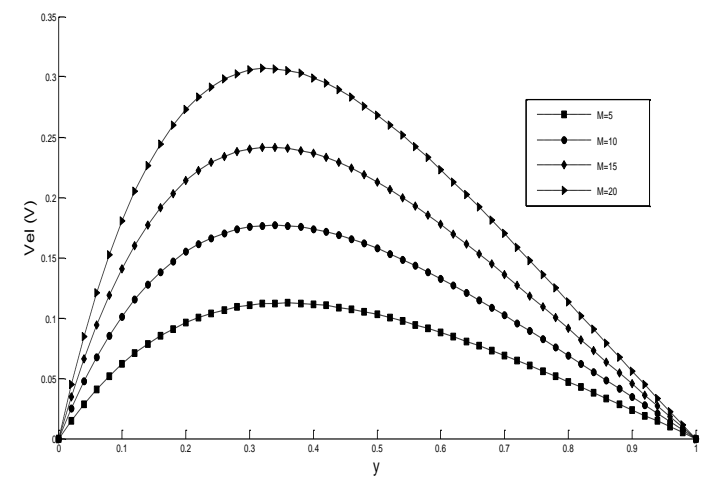

Fig1: Velocity profiles for different values of $\mathrm{M}$. values of $\mathrm{Gr}$.

Figure.1 shows the effect of the magnetic field parameter $M$ on velocity. It is found that the velocity decreases with increasing magnetic parameter. The application of a magnetic field normal to the flow direction has tendency to slow down the movement of the Jeffery fluid because it gives rise to a resistive force called the Lorentz force which acts opposite to the flow direction.

Figure. 2 and 3 analyses the influence of thermal Grashof number Gr in case of cooling of the plate and the solutal Grashof number $\mathrm{G} \varphi$. It is noticed that as $\mathrm{Gr}$ and $\mathrm{G} \varphi$ increases, the velocity profile decreases. To this effect, at higher thermal Grashof number and solutal Grashof number the flow at the boundary is turbulent while at lower the flow at the boundary is laminar.

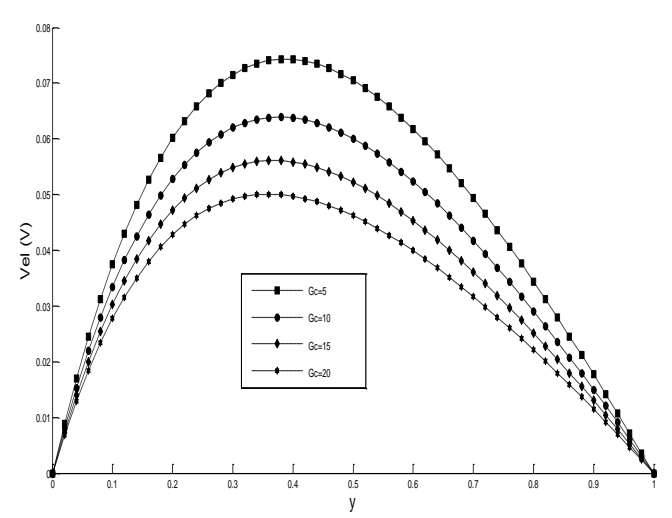

Fig.3: Velocity profiles for different values $\mathrm{G} \varphi$. the Jeffery fluid flow in the porous medium. Throughout the computations, we employ $\mathrm{Gr}=1, \mathrm{G} \varphi$ $=1, \mathrm{M}=2, \mathrm{~K}=1, \mathrm{Sc}=0.22, \mathrm{Kr}=0.5, \mathrm{Sr}=0.05, \mathrm{Du}=0.0001$, $\mathrm{Ec}=0.01, \quad \operatorname{Pr}=7, \quad \omega=1, \quad \lambda=0.5, \quad \lambda 1=0.5, \quad \tau=0.5$, $\mathrm{R}=0.1$ and $\mathrm{Q}=0.1$.
Fig.2: Velocity profiles for different

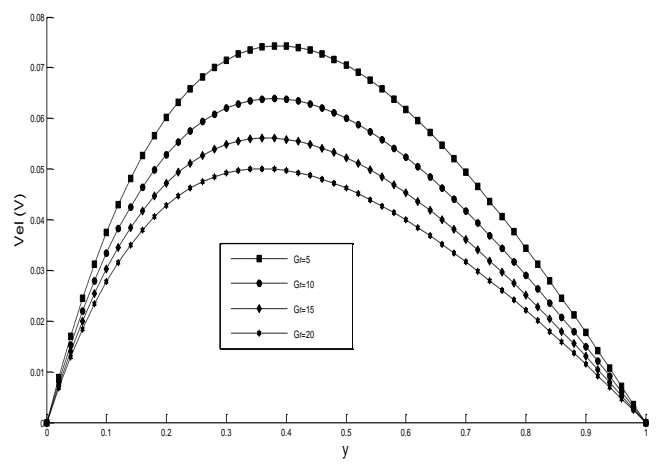

Figure 4 depicts the impact of the permeability parameter $\mathrm{K}$ on the velocity profile. An increase in $\mathrm{K}$ enhances resistant of the porous medium, which will tend to accelerate the flow and increase the velocity of the fluid.

$$
{ }^{*} I D O W U, A S ;{ }^{2} \text { JIMOH, A.; }{ }^{3} \text { AHMED, LO }
$$


Figure 5 and 6 describe the behavior of velocity profiles for different values of real constant and material parameter $\lambda_{1}$. It is evident that the velocity increases with increase in $\lambda_{1}$. Also, the maximum velocity occurs at the centerline of the channel while the minimum at the channel walls. Moreover, the velocity is more of Jeffery fluid $\left(\lambda_{1}>0\right)$ than that of Newtonian $\left(\lambda_{1} \rightarrow 0\right)$. Figure 5 , reveals that increasing in $\lambda$ leads to rise of the velocity profiles.

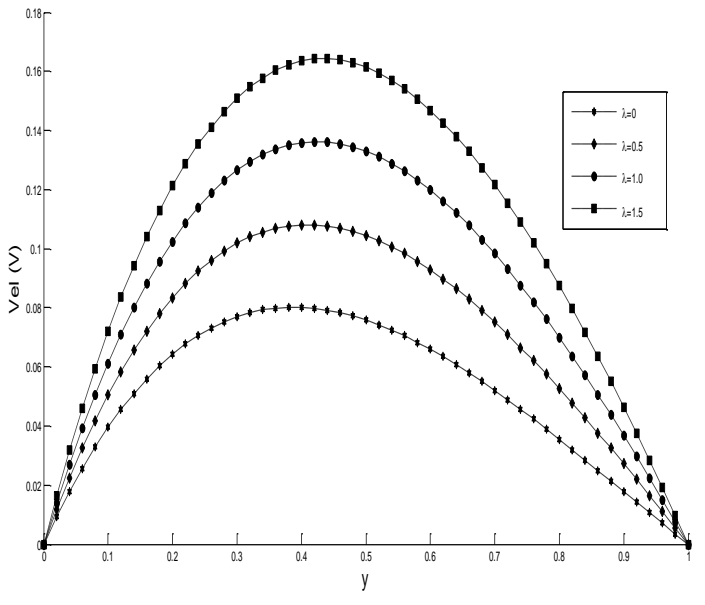

Fig.5.Velocity profiles for different values of $\lambda$.

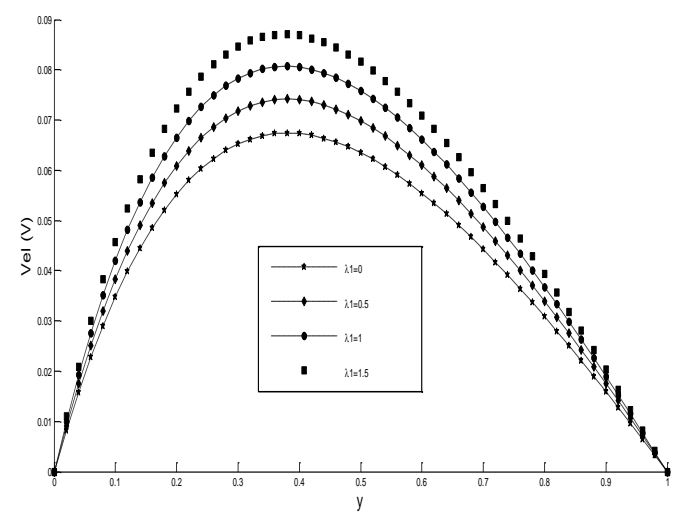

Fig.6. Velocity profiles for different values of $\lambda 1$.

Figure.7. presents typical velocity profiles in the boundary layer for various values of $\omega$. While other parameters are kept at some fixed values, therefore it is observed that enhancement of the oscillatory frequency causes the velocity profiles to fall.

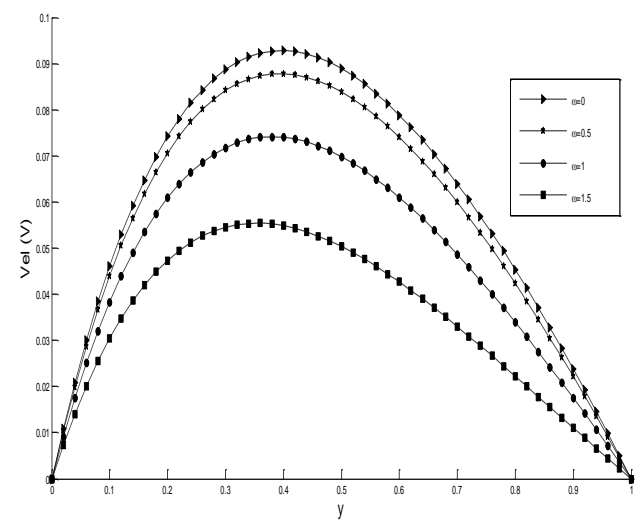

Fig.7. Velocity profiles for different values of $\omega$

Figure 8-10 displays the velocity profiles for different values of Reynolds number Re, radiation parameter $\mathrm{R}$ and Prandtl number Pr. It is observed from figure 8 that an increase in $\mathrm{Re}$, increases the velocity at any point of the fluid. But, figure 9 and 10 illustrate the velocity profiles for different values of the Prandtl number Pr. the Prandtl number defines the ratio of momentum diffusivity to thermal diffusivity. The

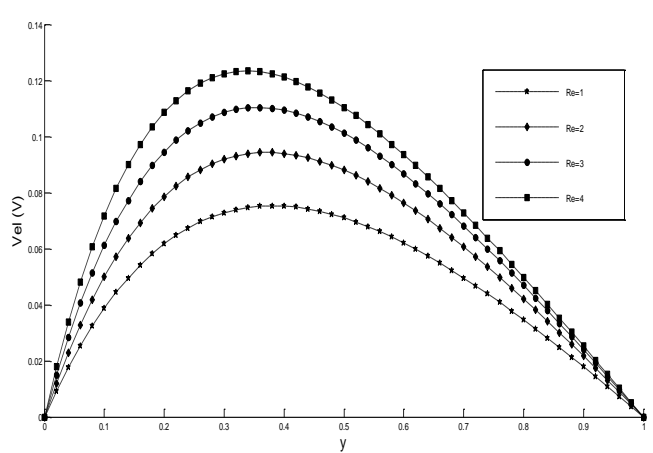

Fig.8 Velocity profiles for different values of Re

numerical results in a decreasing velocity (Figure 10). From Figure 10, it is observed that an increase in the Prandtl number results to a decrease of the velocity profiles. While figure 9 is plotted to show the influence of radiation $\mathrm{R}$, on velocity profiles, this figure displays that increasing the radiation parameter $\mathrm{R}$, decreases the velocity gradients. Figure 11 indicates that, the velocity increases whenever the soret $\mathrm{Sr}$ increased, 
why the velocity decreases whenever the heat source $\mathrm{Q}$ (figure 12) increasing, the presence of heat generation has the tendency to reduce the fluid temperature. This is because The thermal buoyancy effects to decrease in a net reduction in the fluid velocity.

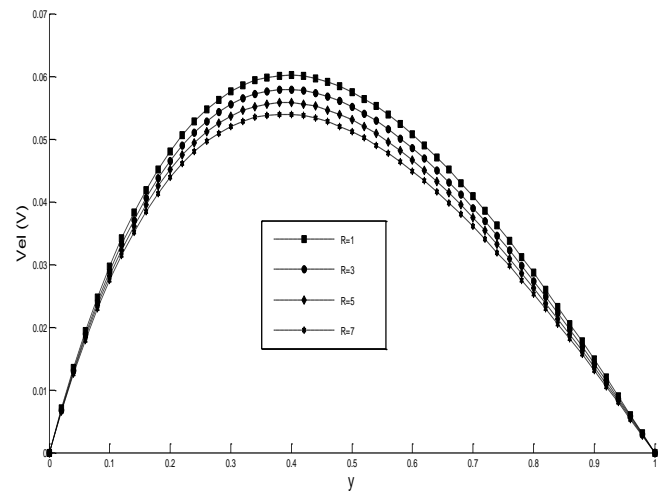

Fig.9. Velocity profiles for different values of R.

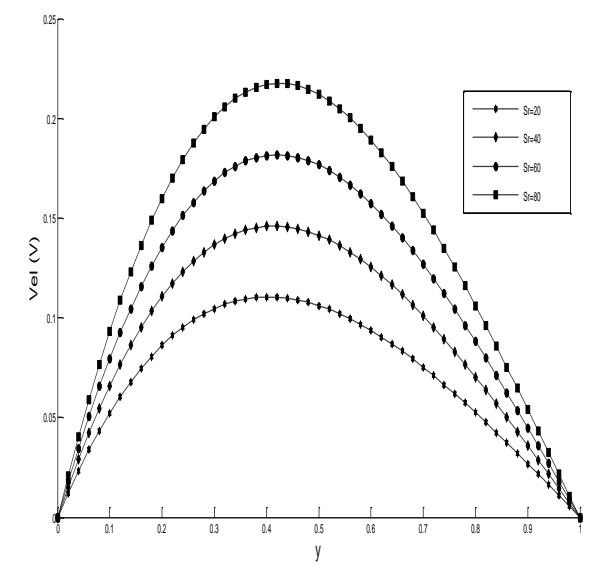

Fig.11. Velocity profiles for different values of $\mathrm{Sr}$

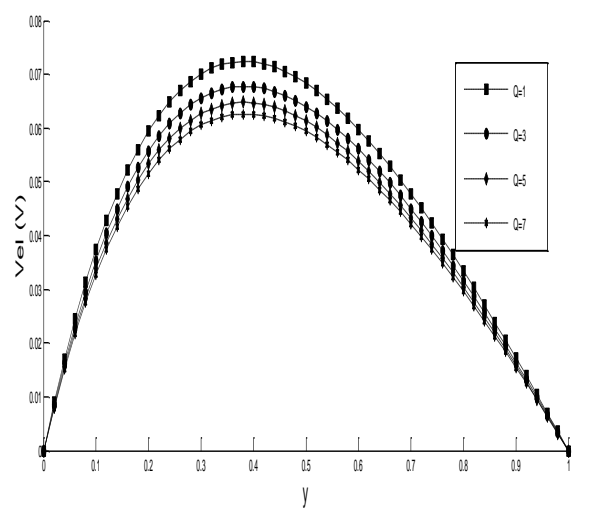

Fig.13 Velocity profiles for different values of Q
The effect of Reynolds number on velocity is shown in figure 8 . It is observed that the velocity decreases with increase in Re.

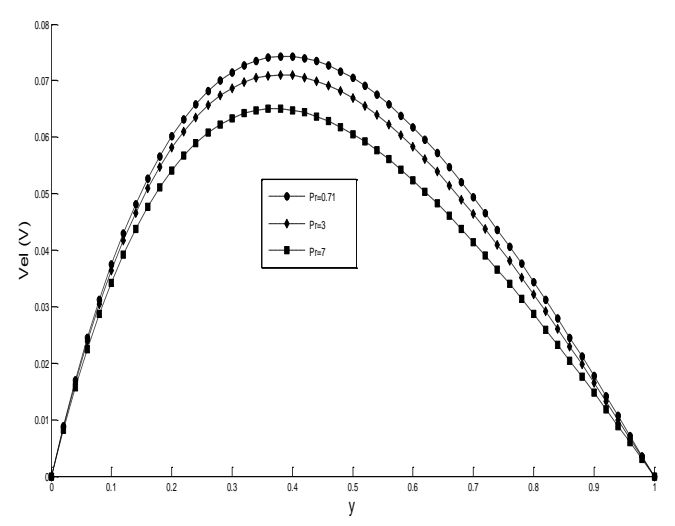

Fig.10. Velocity profiles for different values of Pr.

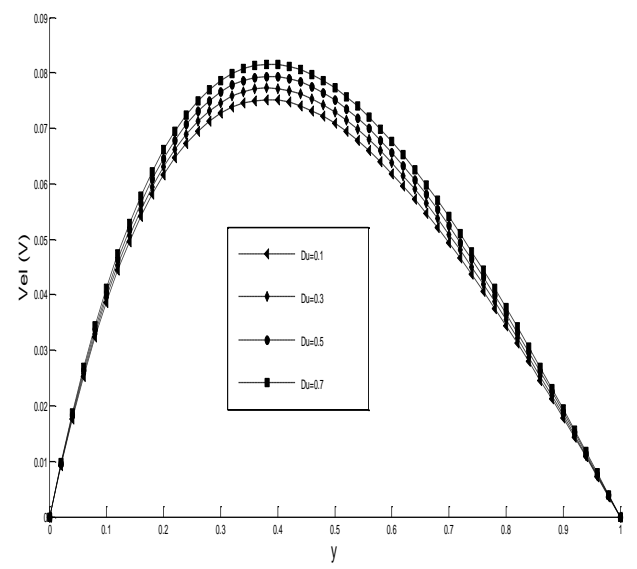

Fig.12. Velocity profiles for different values Du

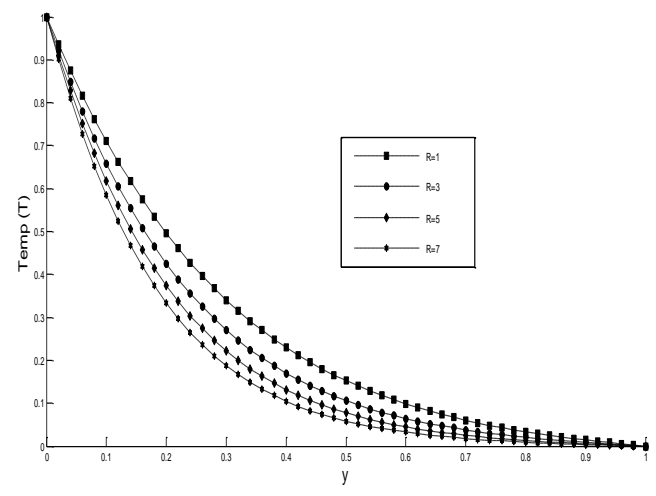

Fig.14. Temperature profiles for different values of $R$ 
Temperature Profiles: The temperature of the flow field suffers a substantial change with the variation of the flow parameters such as radiation R, Prandtl Pr number, heat source $\mathrm{Q}$, Eckert Ec, thermal conductivity parameter $\tau$ and Dufour Du. The relative influence of the various material parameters on the fluid temperature are illustrated in Figures 14- 19.

Figure 14 represent the effects of radiation $\mathrm{R}$, from this figure we observed that increase in the radiation

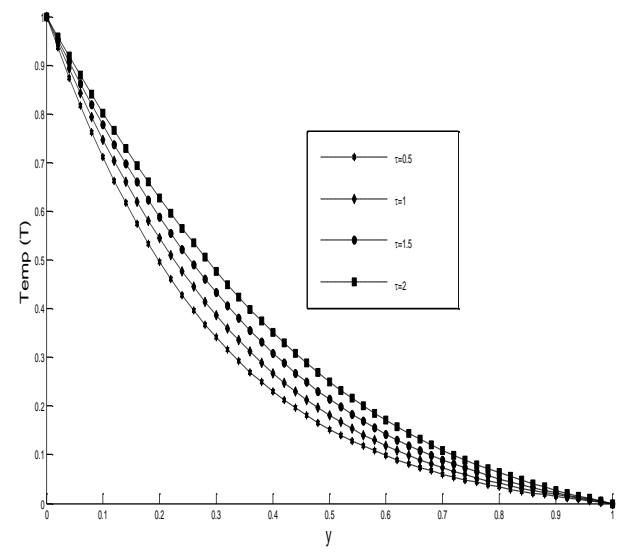

Fig.15. Temperature profiles for different values of $\tau$ parameter decreases the temperature distribution in the thermal boundary layer.

Figure 15 illustrate the variation of temperature profile against span wise coordinate $y$ for various values of thermal conductivity $\tau$ and fixed values of other physical parameter in the presence of variable suction. It is seen clearly that increase in thermal conductivity brings an increase in the temperature of the fluid.

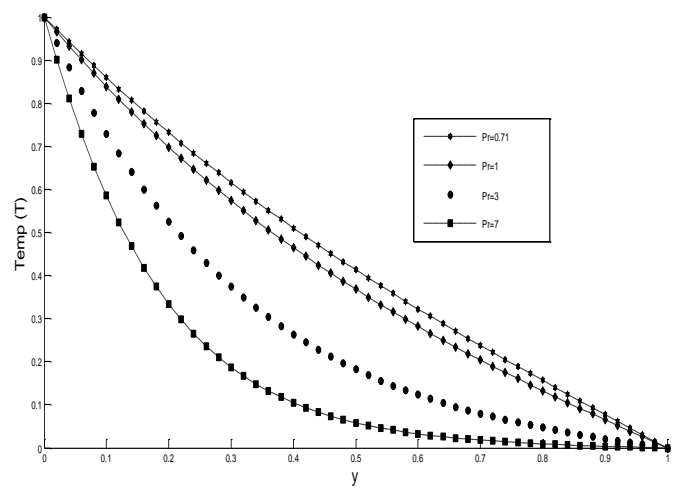

Figure 16 and 17, demonstrates the effect of Prandtl Pr and Eckert Ec number on temperature. It displays that the rate of temperature transfer increases with increasing values of Pr and Ec.

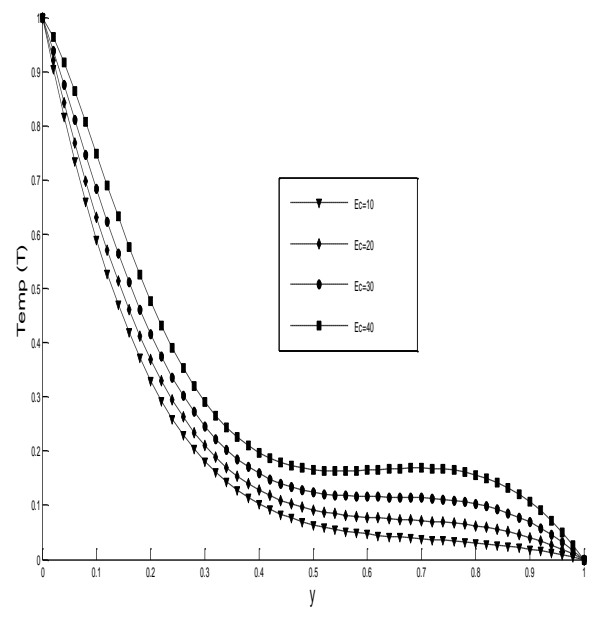

Fig.17. Temperature profiles for different values of Ec $\mathrm{Du}$

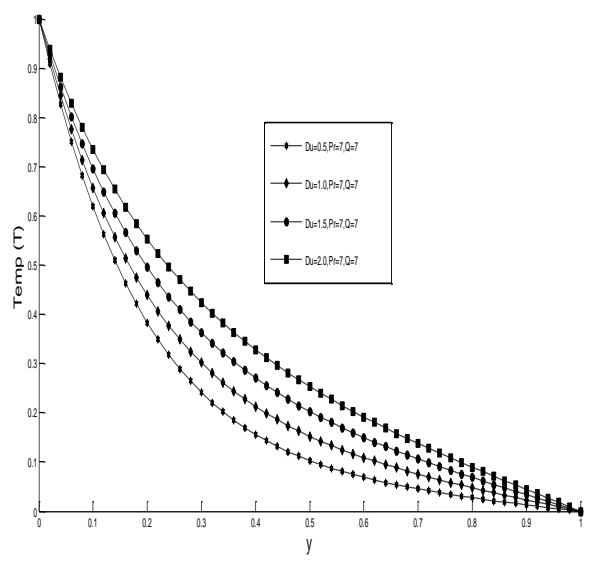

Fig.18. Temperature profiles for different values of

Figures 18 -20 describes the behavior of temperature profiles for different values of Dufour $\mathrm{Du}$,Soret $\mathrm{Sr}$ and heat generation Q. In figure 17-18, it is observed that increasing in Du and $\mathrm{Sr}$ enhances the temperature of the fluid, why figure 18 display the effect of heat generation parameter $Q$ on the temperature decreases with increasing $Q$ as expected. 


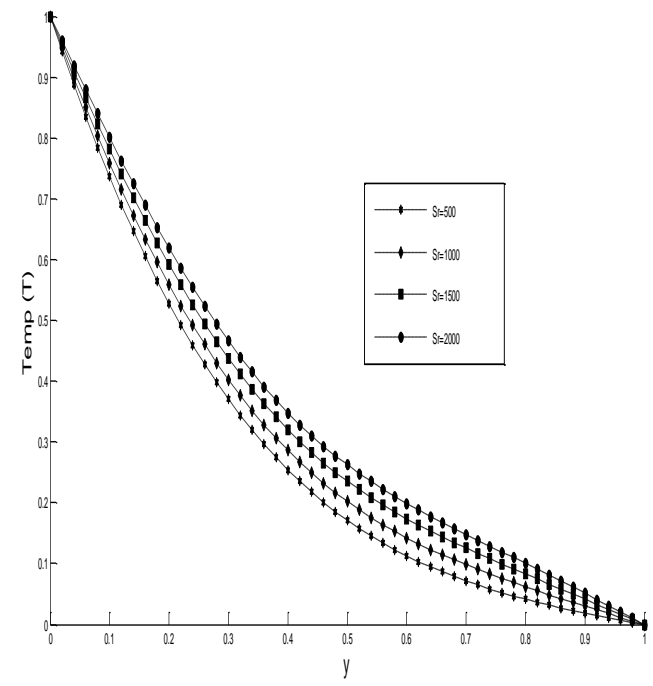

Fig.19. Temperature profiles for different values of $\mathrm{Sr}$ Q

Concentration Profiles: Figures 21-23 graphically show the influence of Schmidt number Sc, Soret number Sr and Chemical reaction $\mathrm{Kr}$. It is found out that an increase in the $\mathrm{Sc}, \mathrm{Sr}$ and $\mathrm{Kr}$ causes a fall in the concentration profiles throughout the boundary layer

Fig.21. Concentration profiles for different values of $\varphi$

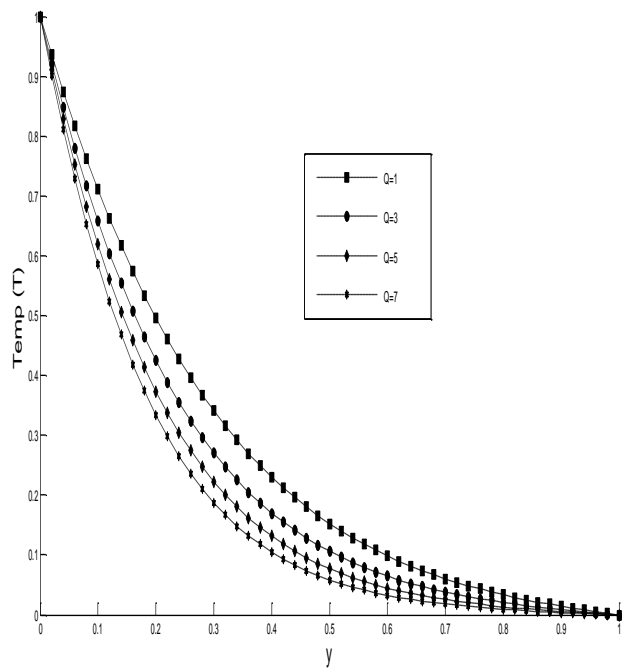

Fig 20 Temperature profiles for different values of

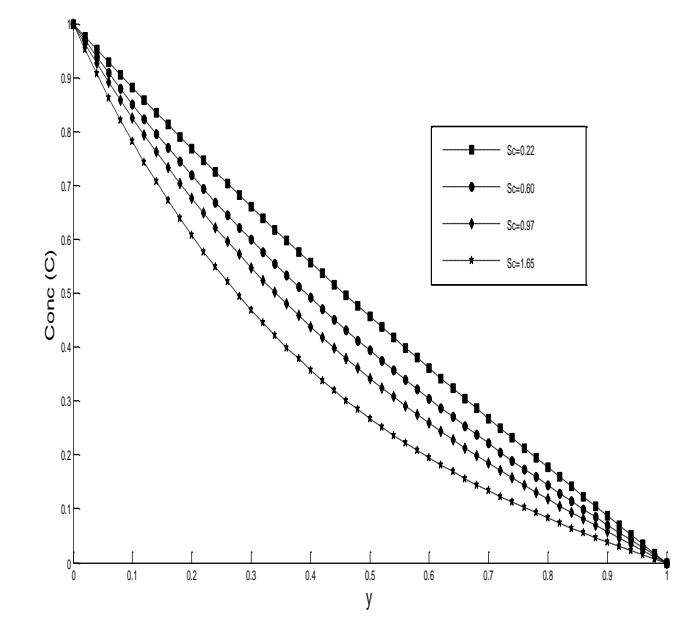

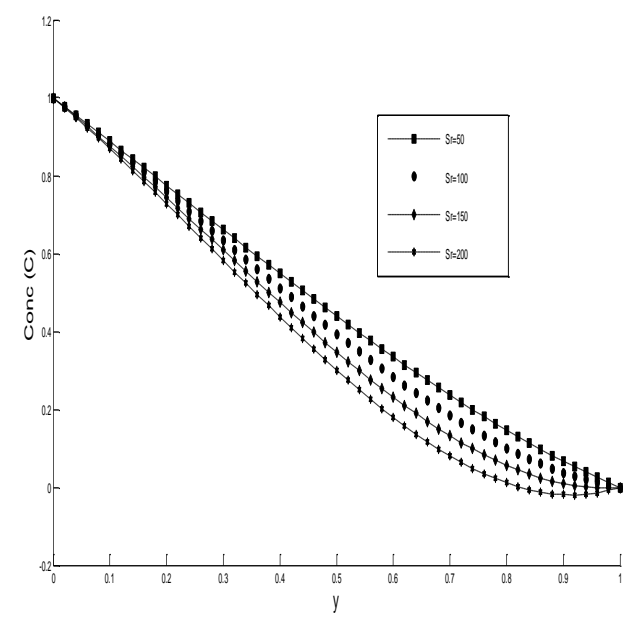

Fig.22. Concentration profiles for different values of $\mathrm{Sr}$ 


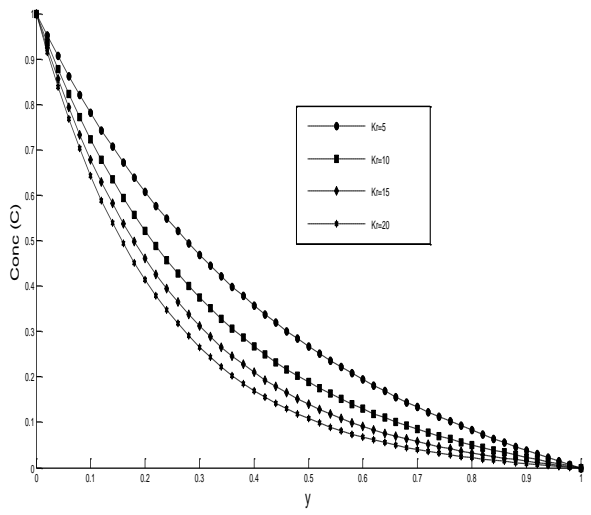

Fig.23. Concentration profiles for different values of $\mathrm{Kr}$

The table below depicts the various parameters; A strong flow circulation is observed when the following parameters were high $\mathrm{M}, \lambda 1, \lambda$. It was discovered that increase in any of the parameters $\mathrm{Gr}, \mathrm{Gc}, \mathrm{K}$, and $\omega$ causes reduction in the Skin friction, It is also seen that as $\tau$ and Ec increases, there is a rise in Nusselt number while an increase in $\mathrm{R}, \mathrm{Pr}, \mathrm{Du}$ and $\mathrm{Q}$, leads to a fall in the Nusselt number, it was noted that as $\mathrm{Kr}, \mathrm{Sr}$ and Sc there are is a fall sherwood number respectively.

The application of thermal conductivity can be found in the satellite industry, which is use to regulate the temperature of the satellite.

TABLE

\begin{tabular}{llll}
\hline $\mathbf{P A R A M E T E R S}$ & SKIN FRICTION & NUSSELT NUMBER & $\begin{array}{l}\text { SHERWOOD } \\
\text { NUMBER }\end{array}$ \\
\hline $\mathbf{M = 5}$ & & & -1.1768 \\
$\mathbf{M}=\mathbf{1 0}$ & 0.7888 & -3.0631 & -1.1769 \\
$\mathbf{M}=\mathbf{1 5}$ & 1.3101 & -3.0579 & -1.1770 \\
$\mathbf{G r = 5}$ & 1.8323 & -3.0503 & -1.1768 \\
$\mathbf{G r}=\mathbf{1 0}$ & 0.4295 & -3.0653 & -1.1768 \\
$\mathbf{G r}=\mathbf{1 5}$ & 0.3867 & -3.0656 & -1.1768 \\
$\mathbf{G c = 5}$ & 0.3548 & -3.0657 & -1.1768 \\
$\mathbf{G c}=\mathbf{1 0}$ & 0.4295 & -3.0653 & -1.1768 \\
$\mathbf{G c = 1 5}$ & 0.3867 & -3.0656 & -1.1768 \\
$\mathbf{K}=\mathbf{1}$ & 0.3548 & -3.0657 & -1.1768 \\
$\mathbf{K}=\mathbf{2}$ & 0.4763 & -3.0650 & -1.1768 \\
$\mathbf{K}=\mathbf{3}$ & 0.3948 & -3.0654 & -1.1768 \\
$\mathbf{P r}=\mathbf{0 . 5}$ & 0.3676 & -3.0656 & -1.2044 \\
$\mathbf{P r}=\mathbf{0 . 7 1}$ & 0.5900 & -1.2717 & -1.2015 \\
$\mathbf{P r}=\mathbf{7}$ & 0.5763 & -1.4574 & -1.1415 \\
$\tau=\mathbf{0}$ & 0.4109 & -5.4039 & -1.1653 \\
$\tau=\mathbf{0 . 5}$ & 0.4578 & -3.8257 & -1.1768 \\
$\tau=\mathbf{1}$ & 0.4763 & -3.0650 & -1.1856 \\
$\mathbf{R = 1}$ & 0.4958 & -2.4852 & -1.1704 \\
$\mathbf{R}=\mathbf{3}$ & 0.4621 & -3.4857 & -1.1589 \\
$\mathbf{R}=\mathbf{5}$ & 0.4389 & -4.2517 & -1.1495 \\
$\mathbf{K r}=\mathbf{5}$ & 0.4228 & -4.8724 & -1.2336 \\
$\mathbf{K r}=\mathbf{1 0}$ & 0.6125 & -0.9528 & -1.3988 \\
$\mathbf{K r}=\mathbf{1 5}$ & 0.6071 & -0.9528 & -1.5539 \\
$\mathbf{S c}=\mathbf{0 . 2 2}$ & 0.6023 & -0.9527 & -1.0634 \\
$\mathbf{S c}=\mathbf{0 . 6 0}$ & 0.4809 & -3.0662 & -1.1768 \\
$\mathbf{S c = 0 . 9 7}$ & 0.4763 & -3.0662 & -1.2917 \\
\hline & 0.4719 & -3.0662 &
\end{tabular}

${ }^{* 1} I D O W U, A S ;{ }^{2}$ JIMOH, A.; ${ }^{3}$ AHMED, LO 


\begin{tabular}{llll}
\hline $\mathbf{S r}=\mathbf{5 0}$ & 0.6046 & -0.9528 & -1.3391 \\
$\mathbf{S r}=\mathbf{1 0 0}$ & 0.5914 & -0.9527 & -1.6035 \\
$\mathbf{S r}=\mathbf{1 5 0}$ & 0.5782 & -0.9527 & -1.8679 \\
$\lambda \mathbf{1 = 0}$ & 0.4312 & -3.0652 & -1.1768 \\
$\lambda \mathbf{1 = 0 . 5}$ & 0.4763 & -3.0650 & -1.1768 \\
$\lambda \mathbf{1 = 1}$ & 0.5234 & -3.0647 & -1.1768 \\
$\lambda=\mathbf{0}$ & 0.3713 & -3.0656 & -1.1768 \\
$\lambda=\mathbf{0 . 5}$ & 0.4763 & -3.0650 & -1.1768 \\
$\lambda=\mathbf{1}$ & 0.5813 & -3.0642 & -1.1768 \\
$\mathbf{E c}=\mathbf{1 0}$ & 0.4196 & -4.9017 & -1.1491 \\
$\mathbf{E c}=\mathbf{2 0}$ & 0.4354 & -4.0377 & -1.1621 \\
$\mathbf{E c}=\mathbf{3 0}$ & 0.4547 & -2.9689 & -1.1782 \\
$\boldsymbol{\omega}=\mathbf{0}$ & 0.5651 & -3.0868 & -1.1780 \\
$\boldsymbol{\omega}=\mathbf{0 . 5}$ & 0.5415 & -3.0810 & -1.1777 \\
$\boldsymbol{\omega}=\mathbf{1}$ & 0.4763 & -3.0650 & -1.1768 \\
$\mathbf{D u}=\mathbf{0 . 1}$ & 0.4806 & -2.9991 & -1.1778 \\
$\mathbf{D u}=\mathbf{0 . 3}$ & 0.4892 & -2.8635 & -1.1779 \\
$\mathbf{D u}=\mathbf{0 . 5}$ & 0.4980 & -2.7227 & -1.1821 \\
$\mathbf{Q}=\mathbf{1}$ & 0.4621 & -3.4857 & -1.1704 \\
$\mathbf{Q}=\mathbf{3}$ & 0.4389 & -4.2517 & -1.1589 \\
$\mathbf{Q}=\mathbf{5}$ & 0.4228 & -4.8724 & -1.1495 \\
& & & \\
\hline
\end{tabular}

Conclusion: We considered the impact of heat and mass transfer on MHD oscillatory flow of Jeffery fluid through a porous medium in a channel in the presence of thermal conductivity, Dufour and Soret in this research. The expressions for the velocity, temperature and concentration are solved numerically by using Crank-Nicolson type. For better understanding of the thermal behavior of this work, however, it may be necessary to perform the experimental works. From the present numerical study the following conclusions were drawn.

(1) Velocity profiles increased due to increase in $\lambda 1, \lambda, \mathrm{M}, \mathrm{Sr}$ and $\mathrm{Du}$, while it decreases due to increase in Gr, Gc, K, Pr, R and $\omega$ (2) An increase in Temperature profile is a function of an increase in $\tau$ and Ec while it decreases due to an increase in R, Pr, Du and Q (3) Concentration profiles decreased due to an increase in $\mathrm{Kr}, \mathrm{Sr}$ and $\mathrm{Sc}$.

\section{REFERENCES}

Bhupendra K. Sharma: Soret and Dufor effect on unsteady MHD mixed convection flow past a radiative vetical porous plate embedded in a porous medium with a chemical reaction. (http://www,

SciRP.org/journal/am). Applied Mathematics, 2012, 3,717-723.

G.Vidyasadar and B.Ramana : Radiation effect on MHD free convection flow of kuvshinshiki fluid with mass transfer past a vertical porous plate through porous medium. Asian Journal of Current Engineering and Maths. 2013 2(3): 170-174.

Idowu, A.S, Joseph, K.M and Daniel .S. : Effect of heat and mass transfer on unsteady MHD oscillatory flow of Jeffery fluid in a horizontal channel with chemical reaction. IOSR: Journal of Mathematics (IOSR-Jm), 2013; 8(5): 74-87

J. Anand Rao, R. Srinivasa Raju and S. Sivaiah: Finite element solution of heat and mass transfer in MHD flow of a viscous fluid past vertical porous plate under oscillatory suction velocity.Journal of applied fluid mechanics 2012, 5(3): 1-10.

K. Srinivasa Rao and P. Koteswara Rao: Effect of heat transfer on MHD oscillatory flow of Jeffery fluid through a porous medium in a tube. International journal of mathematical Archive-3(11), 2012, 46924699; www.ijma.info ISSN: 2229-5046

Kavita. K.: Influence of heat transfer on MHD oscillatory flow of Jeffery fluid in a channel', Adv. Appl. Sci. Res, 2012, 3 (4):2312-2325.

S. Anuradha: Heat and mass transfer on unsteady MHD free convective flow past a semi-infinite vertical plate with soret effect. International journal of science and technoledge, 2014, 2(4) .

I.J Uwanta and E. Omokhuale: Effects variable thermal conductivity on heat and mass transfer with Jeffery fluid. International journal of mathematical Archive; 2014,5(3):135-149. 